\title{
DUALITY AND THE PCF THEORY
}

\author{
SAHARON Shelah AND Jindřich Zapletal
}

\begin{abstract}
We consider natural cardinal invariants $\mathfrak{h}_{\mathfrak{m}}$ and prove several duality theorems, saying roughly: if $I$ is a suitably definable ideal and provably $\operatorname{cov}(\mathrm{I}) \geq$ $\mathfrak{h} \mathfrak{m}_{n}$, then non(I) is provably small. The proofs integrate the determinacy theory, forcing and pcf theory.
\end{abstract}

\section{Introduction}

The authors of [8] considered the following cardinal invariant of the continuum. Let $c:\left[2^{\omega}\right]^{2} \rightarrow 2$ be the partition defined by $c(x, y)=\Delta(x, y) \bmod 2$ where $\Delta(x, y)=\min \{m \in \omega: x(m) \neq y(m)\}$, and let $J$ be the $\sigma$-ideal $\sigma$ generated by the homogeneous sets. It is not difficult to see that $J$ is a proper $\sigma$-ideal; in fact $c$ is the simplest continuous partition of $\left[2^{\omega}\right]^{2}$ with this property. [8] defined the cardinal invariant $\mathfrak{h m}$ as the covering number of the ideal $J$. The invariant $\mathfrak{h m}$ occurs naturaly in several contexts: it is the minimal number of Lipschitz-with-constant- 1 functions from $2^{\omega}$ to itself such that their graphs and graphs of their inverses cover the whole square $2^{\omega} \times 2^{\omega}$. It is also the minimal number of convex subsets necessary to cover a certain closed subset of the Euclidean plane. See [8].

It is consistent with ZFC that $\mathfrak{h m}<\mathfrak{c}$; not surprisingly [9], this is exactly what happens in the Sacks model [8]. On the other hand, $\mathfrak{h m}$ is a very large cardinal invariant in that provably $\mathfrak{h m}^{+} \geq \mathfrak{c}$. Here we prove a duality theorem similar to the one from [9].

Theorem 1.1. Suppose that $I$ is an analytic $\sigma$-ideal such that $Z F C$ proves that $\operatorname{cov}(\mathrm{I}) \geq \mathfrak{h m}$. Then $Z F C$ proves that $\operatorname{non}(\mathrm{I}) \leq \aleph_{3}$.

Here a $\sigma$-ideal is analytic if there is a $\Sigma_{1}^{1}$ set in the plane such that the ideal is $\sigma$-generated by its vertical sections. Similarly, it is possible to define the class of projective ideals. The theorem remains true for projective ideals if the theory ZFC is replaced by ZFC+ "there are $\omega$ many Woodin cardinals".

Received April 16, 2002.

2000 Mathematics Subject Classification. 03E17, 03E04.

The first author was supported by The Israel Science Foundation founded by the Israel Academy of Sciences and Humanities. Publication 791.

The second author was partially supported by grants GA ČR 201-00-1466, NSF DMS0071437, a CLAS UF research award and a visiting appointment at Hebrew University in February and March 2002. 
The theorem remains true if the pair cov, non is replaced by any other dual pair of invariants, such as non, cov or add, cof. However, in order for the various complexity computations to come out right, in these cases we must restrict ourselves to the class of Borel ideals.

The pattern persists to some variations of $\mathfrak{h m}$. For every natural number $n>0$ let $c_{n}:\left[2^{\omega}\right]^{2} \rightarrow n$ be the function defined by $c_{n}(x, y)=\Delta(x, y) \bmod n$ and let $J_{n}$ be the $\sigma$-ideal on $2^{\omega} \sigma$-generated by the sets $X$ such that $c_{n}^{\prime \prime} X \neq n$. Define $J_{1}$ to be the ideal of countable sets. Define $\mathfrak{h m}_{n}$ to be the covering number of the ideal $J_{n}$. It is not difficult to show that $\mathfrak{c}=\mathfrak{h} \mathfrak{m}_{1} \geq \mathfrak{h m}=\mathfrak{h} \mathfrak{m}_{2} \geq \mathfrak{h} \mathfrak{m}_{3} \geq \mathfrak{h} \mathfrak{m}_{4} \geq \ldots$ and $\left(\mathfrak{h m}_{n}\right)^{n-1} \geq \mathfrak{c}$ for every number $n$. Again, $\mathfrak{h m}_{n}$ can be rewritten as the smallest number of functions in a certain class necessary to cover the cube $\left(2^{\omega}\right)^{n}$ with their graphs. We have

Theorem 1.2. Let $n>0$. Suppose that $I$ is an analytic $\sigma$-ideal such that $Z F C$ proves that $\operatorname{cov}(\mathrm{I}) \geq \mathfrak{h m}_{n}$. Then $Z F C$ proves that $\operatorname{non}(\mathrm{I}) \leq \aleph_{n+1}$.

In case of $n=1$ this improves the original duality theorem of [9], and the obtained bound $\aleph_{2}$ is optimal. Finally,

Theorem 1.3. Suppose that $I$ is an analytic ideal such that $Z F C$ proves that $\operatorname{cov}(\mathrm{I}) \geq \min _{n} \mathfrak{h m}_{n}$. Then ZFC proves that non(I) $\leq \aleph_{\omega_{2}+1}$.

The remarks after Theorem 1.1 remain in force for 1.2 and 1.3.

The proofs of the above theorems integrate the effective descriptive theory, determinacy theory, forcing, and pcf theory. This paper contains only the arguments that are specific to the ideals $J_{n}$ in question. The general theory of definable proper forcing is encapsulated into several facts, stated without proof. For the detailed development of this theory, the reader is referred to the monograph [9].

The notation in the paper follows the set theoretic standard of [1]. Whenever $X$ is a set, $x \in X$ its element and $f:\left(2^{\omega}\right)^{X \backslash\{x\}} \rightarrow 2^{\omega}$ is a function, by the graph of the function $f$ we mean the collection $\left\{\vec{r} \in\left(2^{\omega}\right)^{X}: \vec{r}(x)=f(\vec{r} \uparrow X \backslash\{x\})\right\}$. For a $\sigma$-ideal $I$ on the reals, the cardinal $\operatorname{cov}(\mathrm{I})$ and non(I) denote the smallest size of a family of sets in the ideal covering the whole real line, and the smallest size of an $I$-positive set respectively. If $B$ is an $I$-positive set, then $I \uparrow B$ is the ideal generated by $I$ and the complement of $B$.

\section{The forcings associated with $\mathfrak{h m}_{n}$}

The following is a basic simple observation.

Lemma 2.1. Fix a number $n>0$.

1. The ideal $J_{n}$ is $\sigma$-generated by closed sets.

2. The ideal $J_{n}$ is nontrivial, i.e. $2^{\omega} \notin J_{n}$.

As proved in [7], the partition $c_{2}$ is the minimal open partition of $\left[2^{\omega}\right]^{2}$ into two pieces such that the $\sigma$-ideal generated by the homogeneous sets is proper, in the sense that if $d$ is any other such a partition then there is a Borel injection 
$i: 2^{\omega} \rightarrow 2^{\omega}$ such that for all $x \neq y \in 2^{\omega} d(i(x), i(y))=c_{2}(x, y)$. Similar minimality result holds true for the partitions $c_{n}$.

Proof. The partitions $c_{n}$ are continuous. Therefore, if $X \subset 2^{\omega}$ is a generating set of the ideal $J_{n}$ (the image $c_{n}^{\prime \prime}[X]^{2}$ does not contain some number $m \in n$ ), then even its closure is such a generating set of the ideal $J_{n}$ (its image under $c_{n}$ still does not contain that number $m$ ). The first item immediately follows.

For the second item we will just show that the ideal $J_{n}$ is a subideal of the meager ideal, for every number $n>0$. Fix the number $n$ and let $X \subset 2^{\omega}$ be a closed generating set of the ideal $J_{n}$ so that the image $c_{n}^{\prime \prime}[X]^{2}$ leaves out some number $m \in n$. It will be enough to show that the set $X$ is nowhere dense. And indeed, if $s \in 2^{<\omega}$ is a finite sequence representing some basic open set, prolong it to obtain a sequence $t \supset s$ whose length is equal to $m$ modulo $n$. By the choice of the set $X$, for one of the sequences $t^{\frown} 0, t^{\frown} 1$ the set $X$ contains no infinite binary sequences extending it. Thus the set $X$ is nowhere dense as desired.

Now look at the partial order $P_{n}$ of $J_{n}$-positive Borel sets ordered by inclusion. By Lemma 1.2 and 1.3 of [10], this poset adds a single real which falls out of all $J_{n}$-small sets and it is proper. In fact, this partial order has a natural combinatorially simple dense subset. Call a tree $T \subset 2^{<\omega} n$-fat if it is nonempty and for every node $s \in T$ and every number $m \in n$ there is a splitnode $t \supset s$ in the tree $T$ whose length is equal to $m$ modulo $n$.

Lemma 2.2. If $T$ is an $n$-fat tree then $[T] \notin J_{n}$. Moreover, if $A \subset 2^{\omega}$ is an analytic set, then either it contains a subset of the form $[T]$ for some $n$-fat tree, or else it belongs to the ideal $J_{n}$.

Proof. The regularity property of analytic sets can be proved in several ways; we give a classical determinacy argument following the proof of the perfect set theorem. Suppose that $A \subset 2^{\omega}$ is a set. Consider the game $G_{n}(A)$ where players Adam and Eve alternate to play finite binary sequences $s_{0}, s_{1}, \ldots$ and bits $b_{0}, b_{1}, \ldots$ respectively such that Adam's sequences $s_{i}$ form an extension increasing chain such that $\widehat{s_{i}} b_{i} \subset s_{i+1}$ and the length of the sequence $s_{i}$ is equal to $i$ modulo $n$. Adam wins if $\bigcup_{i} s_{i} \in A$.

First, Adam has a winning strategy in the game $G_{n}(A)$ if and only if the set $A$ contains all branches of some $n$-fat tree. If $\sigma$ is a winning strategy for Adam then the downward closure of the set of all sequences that can arise in a play according to the strategy $\sigma$ is an $n$-fat tree and all of its branches belong to the set $A$. On the other hand, if $[T] \subset A$ is an $n$-fat tree then Adam can easily win the game $G_{n}(A)$ by playing only splitnodes in the tree $T$.

Second, Eve has a winning strategy if and only if the set $A$ belongs to the ideal $J_{n}$. If $A \subset \bigcup_{i} X_{i}$ is in the ideal $J_{n}$, covered by countably many sets $X_{i}$ such that $i \bmod n \notin c_{n}^{\prime \prime}\left[X_{i}\right]$, then Eve wins by answering Adam's sequence $s_{i}$ in the $i$-th round with a bit $b_{i}$ such that no element of the set $X_{i}$ begins with $s_{i} b_{i}$. There is such a bit $b_{i}$ because no two elements of the set $X_{i}$ can first differ at the number $i \bmod n$ which is the length of the sequence $s_{i}$. Of course, if Eve plays 
in this way she will win in the end, since the real $\bigcup_{i} s_{i}$ will fall out of all the sets $X_{i}$ and therefore out of the set $A$. On the other hand, if Eve has a winning strategy $\sigma$ then the set $A$ is in the ideal $J_{n}$. For every position $p$ consistent with the strategy $\sigma$ which ends after the round $i_{p}$ was completed with some finite binary sequence $t_{p}$, let $S_{p}$ be the downward closure of the set $\left\{t \in 2^{<\omega}\right.$ : for no finite sequence $s$ and no bit $b$ it is the case that the play $p^{\wedge} s^{\wedge} b$ observes all the rules and the strategy $\sigma$ and $\left.t_{p}^{\frown} s^{\frown} b \subset t\right\}$. It is not difficult to verify that the closed set $\left[S_{p}\right]$ is in the ideal $J_{n}$ since $i_{p} \bmod n \notin c_{n}^{\prime \prime}\left[\left[S_{p}\right]\right]^{2}$. We also have that $A \subset \cup_{p}\left[S_{p}\right]$ : if some infinite binary sequence $x \in A$ fell out of all the sets $\left[S_{p}\right]$, then a play of the game observing the strategy $\sigma$ could be constructed such that the resulting sequence is just $x$ and thus Adam won, contradicting the assumption that $\sigma$ was a winning strategy for Eve.

The previous two paragraphs together with the classical determinacy results of [3] show that Borel sets have the regularity property, and that $\Sigma_{n}^{1}$ determinacy implies that $\Sigma_{n}^{1}$ sets have the regularity property. A standard trick described for example in [2] can be used to reduce the assumption to $\Delta_{n}^{1}$ determinacy for the regularity of $\Sigma_{n}^{1}$ sets, proving the Lemma.

Thus the partial order $P_{n}$ is in forcing sense equivalent to the ordering of $n$-fat trees under inclusion. It is now possible to give a detailed analysis of its forcing properties, using standard combinatorial methods. However, the approach of the current paper is completely different. We shall need only the following consequence:

Corollary 2.3. The ideal $J_{n}$ is homogeneous. The forcing $P_{n}$ is homogeneous.

Here,

Definition 2.4. [9] A $\sigma$-ideal $I$ on $2^{\omega}$ is called homogeneous if for every $I$ positive Borel set $B$ there is a function $f: 2^{\omega} \rightarrow B$ such that the preimages of $I$-small sets are $I$-small.

Homogeneity of an ideal $I$ is a convenient way of securing the equalities $\operatorname{cov}(\mathrm{I})=\operatorname{cov}(\mathrm{I} \uparrow \mathrm{B})$ and $\operatorname{non}(\mathrm{I})=\operatorname{non}(\mathrm{I} \uparrow \mathrm{B})$ for an arbitrary positive Borel set $B$. If $X$ is a collection of $I$-small sets covering the set $B$ then the collection of their $I$-small preimages covers the whole $2^{\omega}$; it has size $\leq|X|$. And if $Y \subset 2^{\omega}$ is an $I$-positive set, then its $f$-image is an $I$-positive subset of the set $B$; it has size $\leq|Y|$. The key property of homogeneity is that it is frequently preserved under the iterated Fubini powers of the ideal-[9].

Proof. Suppose that $B \subset 2^{\omega}$ is a $J_{n}$-positive Borel set, and let $i: 2^{\omega} \rightarrow B$ be a continuous injection respecting the partition $c_{n}$; such an injection exists by the previous lemma. Clearly, the preimages of $J_{n}$-small sets must be $J_{n}$-small, and therefore the ideal $J_{n}$ is homogeneous. The function $\pi$ defined by $\pi(C)=\pi^{\prime \prime} C$ for every $J_{n}$-positive Borel set $C \subset 2^{\omega}$, is an isomorphism between the poset $P_{n}$ and $P_{n}$ below the condition $\pi\left(2^{\omega}\right) \subset B$. Note that Borel injective images of Borel sets are Borel. 
Corollary 2.5. The ideal $J_{n}$ is $\Pi_{1}^{1}$ on $\Sigma_{1}^{1}$.

Proof. Every $\sigma$-ideal $K$ such that the poset $\mathcal{B}\left(2^{\omega}\right) \backslash K$ ordered by inclusion is bounding and contains a $\Sigma_{1}^{1}$ collection of compact sets as a dense subset, is $\Pi_{1}^{1}$ on $\Sigma_{1}^{1}$-see the appendix of [9]. A direct proof along the lines of [2] is also possible.

Using Theorem 7.4 of [9] and the previous lemmas it is also possible to conclude that the countable support iteration of $P_{n}$ forcings is the optimal way to increase the invariant $\mathfrak{h m}_{n}$. While this is an interesting fact in itself, it plays no role in the proofs of the theorems from the introduction.

Corollary 2.6. $(Z F C+L C)$ Suppose $n>0$ and $\mathfrak{x}$ is a tame invariant. If $\mathfrak{x}<$ $\mathfrak{h m}_{n}$ holds in some forcing extension, then it holds in the countable support iterated $P_{n}$-extension.

As an aside, let us define the cardinal invariant $\mathfrak{h m}_{\omega}$ in the following way. Let $\left\{a_{n}: n \in \omega\right\}$ be an arbitrary partition of $\omega$ into infinite sets, and for distinct sequences $x, y \in 2^{\omega}$ define $c_{\omega}(x, y)=n$ if the smallest number $m$ such that $x(m) \neq y(m)$ belongs to the set $a_{n}$. Let $J_{\omega}$ be the $\sigma$-ideal generated by the sets $X \subset 2^{\omega}$ such that $c_{\omega}^{\prime \prime} X \neq \omega$, and define $\mathfrak{h m}_{\omega}$ to be the covering number of this ideal. The invariant $\mathfrak{h m}_{\omega}$ is independent of the initial choice of the partition $\left\{a_{n}: n \in \omega\right\}$ : if $\left\{a_{n}^{\prime}: n \in \omega\right\}$ is another such a partition, $c_{\omega}^{\prime}$ the associated function and $\mathfrak{h m}_{\omega}^{\prime}$ the associated invariant, it is not difficult to find a continuous injection $i: 2^{\omega} \rightarrow 2^{\omega}$ reducing the function $c_{\omega}$ to $c_{\omega}^{\prime}$ in the sense that $c_{\omega}^{\prime}(i(x), i(y))=c_{\omega}(x, y)$ for every two distinct sequences $x, y \in 2^{\omega}$. This shows that $\mathfrak{h m}_{\omega} \leq \mathfrak{h m}_{\omega}^{\prime}$, and by symmetricity $\mathfrak{h m}_{\omega}=\mathfrak{h m}_{\omega}^{\prime}$. All the previous results all apply to the case of the invariant $\mathfrak{h m}_{\omega}$. However, it is impossible to generalize the theorems stated in the introduction to the case of $\mathfrak{h m}_{\omega}$, since the results of the next section will not be applicable.

\section{The iterated Fubini powers of the ideals $J_{n}$}

The theorems stated in the introduction are proved using a careful analysis of the countable support iteration of the forcings $P_{n}$. We will need to find an upper bound, in terms of the $\aleph$ function, of the uniformities of the iterated Fubini powers of the ideals $J_{n}$. Recall:

Definition 3.1. [9] Suppose that $I$ is a $\sigma$-ideal on the real line and $\alpha \in \omega_{1}$ is an ordinal. The $\alpha$-th iterated Fubini power of the ideal $I$ is the $\sigma$-ideal $I^{\alpha}$ on $\mathbb{R}^{\alpha}$ consisting of those sets $A \subset \mathbb{R}^{\alpha}$ for which Adam has a winning strategy in the two person game $G(A)$ of length $\alpha$. In $\beta$-th round of the game $G(A)$ first Adam plays a set in the ideal $I$ and then Eve chooses a real not in the set. Eve wins if the sequence of her answers belongs to the set $A$.

But in fact, in order to streamline the notation, we will not deal with the ideals $J_{n}$ directly. For every $n>0$ let $K_{n}$ be the $\sigma$-ideal on $\left(2^{\omega}\right)^{n}$ generated by the graphs of Borel partial functions from $\left(2^{\omega}\right)^{n \backslash\{k\}}$ to $2^{\omega}$ for all $k \in n$. There is 
a natural relationship between the ideals $K_{n}$ and $J_{n}$ : consider the bijection $g_{n}$ between $\left(2^{\omega}\right)^{n}$ and $2^{\omega}$ defined by $g_{n}\left(x_{0}, x_{1}, \ldots x_{n-1}\right)=y$ where $y(m)=x_{l}(k)$ where $k$ is the integer part of $m / n$ and $l=m \bmod n$. What is the preimage of the ideal $J_{n}$ ? Let $X \subset 2^{\omega}$ be a closed set such that $c_{n}^{\prime \prime} X$ misses at least one value, say $k \in n$. It is not difficult to see that the preimage of the set $X$ under the bijection $g$ is a graph of a partial Borel function from $\left(2^{\omega}\right)^{n \backslash\{k\}}$ to $2^{\omega}$ : if $\vec{x}=\vec{y}$ are two $n$-tuples such that $g_{n}(\vec{x}), g_{n}(\vec{y}) \in X$ and $\vec{x}(m)=\vec{y}(m)$ for all numbers $m \in n$ different from $k$, then necessarily $\vec{x}(k)=\vec{y}(k)$ as well, since otherwise $c_{n}\left(g_{n}(\vec{x}), g_{n}(\vec{y})\right)=k$, contradicting the choice of the set $X$. Thus clearly $\left\{g^{-} 1(X): X \in J_{n}\right\} \subset K_{n}, \operatorname{non}\left(\mathrm{J}_{n}\right) \leq \operatorname{non}\left(\mathrm{K}_{n}\right)$ and for each countable ordinal $\alpha, \operatorname{non}\left(J_{n}^{\alpha}\right) \leq \operatorname{non}\left(\mathrm{K}_{n}^{\alpha}\right)$. Thus it will be enough to find the uniformities of the ideals $K_{n}^{\alpha}$.

Lemma 3.2. Let $n>0$ be a natural number and $\alpha$ be a countable ordinal. Then $\operatorname{non}\left(\mathrm{K}_{n}^{\alpha}\right) \leq \aleph_{n+1}$.

Proof. The following is the key fact in the argument:

Fact 3.3 (Shelah). Let $\kappa>\omega_{1}$ be a regular cardinal. For every countable limit ordinal $\alpha \in \omega_{1}$ there is a sequence $\vec{C}=\left\langle C_{\delta}: \delta \in \kappa\right\rangle$ such that

1. $C_{\delta} \subset \delta$ is a closed set of ordertype $\leq \alpha$

2. for every ordinal $\delta \in \kappa$ and every accumulation point $\gamma \in C_{\delta}$, the set $C_{\gamma}$ is just $C_{\delta} \cap \gamma$

3. for every closed unbounded set $E \subset \kappa$ there is an ordinal $\delta \in E$ such that the set $C_{\delta}$ is cofinal in $\delta$ of ordertype $\alpha$ and it is a subset of $E$.

This Fact was announced in [5], page 136, remark 2.14A. The proof is in [4], available from the Mathematics ArXiv. Now fix a number $n>0$ and a countable ordinal $\alpha \in \omega_{1}$, without loss assuming that $\alpha$ is limit. For every number $m \in n$ use the Fact to choose a club guessing sequence $\vec{C}_{m}=\left\langle C_{\delta}^{m}: \delta \in \omega_{m+2}\right\rangle$ on $\omega_{m+2}$. We may certainly assume that $\mathfrak{c} \geq \aleph_{n+1}$ and so we can choose a sequence $\vec{s}=\left\langle s_{\delta}: \delta \in \omega_{n+1}\right\rangle$ of pairwise distinct reals-elements of $2^{\omega}$. For every $n$-tuple $\vec{\delta} \in \prod_{m \in n} \omega_{m+2}$ such that the sets $C_{\vec{\delta}(m)}^{m}$ have ordertype $\alpha$, let $\langle\vec{\delta}(m)(\beta): \beta \in \alpha\rangle$ enumerate the nonaccumulation points of these sets in the increasing order, and let $\vec{r}_{\vec{\delta}} \in\left(\left(\left(2^{\omega}\right)^{n}\right)^{\alpha}\right)$ be the $\alpha$-sequence whose $\beta$-th element is the point $\left\langle s_{\vec{\delta}(m)(\beta)}: m \in n\right\rangle$ in the space $\left(2^{\omega}\right)^{n}$. It will be enough to show that the set $\left\{\vec{r}_{\vec{\delta}}: \vec{\delta} \in \prod_{m \in n} \omega_{m+2}\right\}$ is $J_{n}^{\alpha}$-positive.

To prove this, for every Adam's strategy $\sigma$ in the transfinite game we need to find an $n$-tuple $\vec{\delta}$ such that the sequence $\vec{r}_{\vec{\delta}}$ is a legal counterplay against the strategy $\sigma$. So fix the strategy $\sigma$ and by downward induction on $m \in n$ find

1. a continuous increasing $\in$-tower $\vec{M}_{m}=\left\langle M_{\delta}^{m}: \delta \in \omega_{m+2}\right\rangle$ of elementary submodels of a large enough structure, each of them of size $\aleph_{m+1}$ and such that $M_{\delta}^{m} \cap \omega_{m+2} \in \omega_{m+2}$. In particular, we require $\vec{C}_{k}: k \in n+1, \vec{s}, \sigma \in$ $M_{0}^{m}$. Let $E_{m}=\left\{\delta \in \omega_{m+2}: M_{\delta}^{m} \cap \omega_{m+2}=\delta\right\}$; this is a closed unbounded subset of $\omega_{m+2}$. 
2. an ordinal $\delta_{m} \in \omega_{m+2}$ such that the set $C_{\delta_{m}}^{m} \subset \delta_{m}$ is cofinal of ordertype $\alpha$ and it is a subset of the club $E_{m}$. For every two numbers $k \in m \in n$ we demand that $\delta_{m} \in M_{0}^{k}$.

Let $\vec{\delta}=\left\langle\delta_{m}: m \in n\right\rangle$. We claim that the sequence $\vec{r}_{\vec{\delta}}$ is the desired legal counterplay against the strategy $\sigma$. So look at an arbitrary round $\beta \in \alpha$ and suppose that the sequence does constitute a legal counterplay up to this point. What happens at round $\beta$ ?

The important observation is that for every integer $k \in n$, the play up to this round is in the model $M_{\vec{\delta}(k)(\beta)}^{k}$ since it is defined from objects that belong to this model. In particular, one of the parameters in the definition is the set $C_{\vec{\delta}(k)}^{k} \cap \vec{\delta}(k)(\beta)$ which is in the model by the coherence requirement (2) in Fact 3.3.

The strategy $\sigma$ now commands Adam to play partial Borel functions $\left\{f_{m k}\right.$ : $k \in n, m \in \omega\}$ such that $f_{m k}$ is a function from $\left(2^{\omega}\right)^{n \backslash\{k\}}$ to $2^{\omega}$. We must show that the point $\vec{r}_{\vec{\delta}}(\beta)$ is not contained in the graph of any of these functions. So choose integers $m \in \omega$ and $k \in n$. Consider the set $Y=\left\{f_{m k}(\vec{u}, \vec{v}): \vec{u} \in\left(2^{\omega}\right)^{k}\right.$ is a sequence all of whose entries are on the $\vec{s}$-sequence, indexed by ordinals smaller than $\omega_{k+1}$ and $\vec{v} \in\left(2^{\omega}\right)^{n \backslash(k+1)}$ is a sequence all of whose entries are on the $\vec{s}$-sequence, indexed by ordinals in the set $\left.\bigcup_{k+2 \in l \in n+2} C_{\vec{\delta}(l)}^{l}\right\}$. This set is of size $<\omega_{k+2}$ and it belongs to the model $M_{\vec{\delta}(k)(\beta)}^{k}$. Thus $Y \subset M_{\vec{\delta}(k)(\beta)}^{k}$, in particular $s_{\vec{\delta}(k)(\beta)} \notin Y$, which by the definition of the set $Y$ means that the point $\vec{r}_{\vec{\delta}}(\beta)=\left\langle s_{\vec{\delta}(l)(\beta)}: l \in n\right\rangle$ is not on the graph of the function $f_{m k}$ as desired. The lemma follows.

Corollary 3.4. Let $n>0$ be a natural number and let $\alpha$ be a countable ordinal. $\operatorname{non}\left(\mathrm{J}_{n}^{\alpha}\right) \leq \aleph_{n+1}$.

In order to analyse the countable support iteration in which the forcings $P_{n}$ alternate, we need to change the approach a little bit:

Definition 3.5. Suppose that $\vec{I}$ is an $\omega$-sequence of $\sigma$-ideals on the reals and $\alpha$ is a countable ordinal. The ideal $\vec{I}^{\alpha}$ on $\mathbb{R}^{\omega \cdot \alpha}$ consists of those sets $A \subset \mathbb{R}^{\omega \cdot \alpha}$ for which Adam has a winning strategy in the transfinite two person game $G(A)$ of length $\omega \cdot \alpha$. In the $\omega \cdot \beta+n$-th round of this game, Adam plays a set in the ideal $\vec{I}(n)$ and Eve responds with a real which is not in this set. Eve wins if the sequence of her answers belongs to the set $A$.

Let $\vec{K}$ be the $\omega$-sequence of ideals given by $\vec{K}(n)=K_{n+1}$.

Lemma 3.6. Let $\alpha$ be a countable ordinal. Then $\operatorname{non}\left(\overrightarrow{\mathrm{K}}^{\alpha}\right) \leq \aleph_{\omega_{2}+1}$.

Proof. Let $\alpha$ be an arbitrary countable ordinal. First we need to fix several objects whose existence is provable in ZFC.

1. An increasing sequence $\vec{\kappa}=\left\langle\kappa_{\delta}: \delta \in \omega_{2}\right\rangle$ of regular cardinals below $\aleph_{\omega_{2}}$ such that the true cofinality of their product modulo the bounded ideal on $\omega_{2}$ is $\omega_{\omega_{2}+1}$, from [6], Chapter II, Theorem 1.5. This means that there is 
a sequence $\vec{h}=\left\langle h_{\gamma}: \gamma \in \omega_{\omega_{2}+1}\right\rangle$ of functions in $\prod_{\delta} \kappa_{\delta}$ which is increasing and cofinal in the modulo bounded ordering. Fix such a sequence.

2. A club guessing sequence $\vec{C}=\left\langle C_{\gamma}: \gamma \in \omega_{\omega_{2}+1}\right\rangle$ from Fact 3.3. The sequence will guess closed unbounded subsets of $\omega_{\omega_{2}+1}$ by segments of length $\omega \cdot \omega \cdot \alpha$. For every ordinal $\gamma \in \omega_{\omega_{2}+1}$ let $C(\gamma, \beta)$ denote the $\beta$-th nonaccumulation point of the set $C_{\gamma}$.

3. A club guessing sequence $\vec{D}=\left\langle D_{\delta}: \delta \in \omega_{2}\right\rangle$ from Fact 3.3. The sequence will guess closed unbounded subsets of $\omega_{2}$ by segments of length $\omega \cdot \omega \cdot \alpha$ again, with similar notational convention for as in the previous item, using the symbol $D(\delta, \beta)$ for the $\beta$-th nonaccumulation point of the set $D_{\delta}$.

4. Without harm we may assume that $\mathfrak{c}>\aleph_{\omega_{2}}$. So let us fix a sequence $\vec{s}=\left\langle s_{\gamma}: \gamma \in \omega_{\omega_{2}+1}\right\rangle$ of pairwise distinct reals (elements of $2^{\omega}$ ).

Now suppose that $\gamma \in \omega_{\omega_{2}+1}$ and $\delta \in \omega_{2}$ are ordinals such that the ordertypes of the sets $C_{\gamma}$ and $D_{\delta}$ are both $\omega \cdot \omega \cdot \alpha$. Define a $\omega \cdot \alpha$ sequence $\vec{r}_{\gamma \delta}$ by setting its $\omega \cdot \beta+n$-th element to be the point in the space $\left(2^{\omega}\right)^{n+1}$ whose $k$-th coordinate for every number $k \in n+1$ is the real number on the $s$ sequence indexed by the ordinal $h_{C(\gamma, \omega \cdot(\omega \cdot \beta+n)+k)}(D(\delta, \omega \cdot(\omega \cdot \beta+n)+n-k))$. We will show that the collection $\left\{\vec{r}_{\gamma \delta}: \gamma \in \omega_{\omega_{2}+1}, \delta \in \omega_{2}\right\}$ is $\vec{K}^{\alpha}$-positive, proving the lemma. This means that for every Adam's strategy $\sigma$ in the transfinite game we must find ordinals $\gamma \in \omega_{\omega_{2}+1}$ and $\delta \in \omega_{2}$ such that the sequence $\vec{r}_{\gamma \delta}$ is a legal counterplay against the strategy.

Fix a continuous increasing $\in$-tower $\left\langle M_{\gamma}: \gamma \in \omega_{\omega_{2}+1}\right\rangle$ of elementary submodels of large enough structure, each of them of size $\aleph_{\omega_{2}}$ and such that $M_{\gamma} \cap \omega_{\omega_{2}+1} \in$ $\omega_{\omega_{2}+1}$. In particular, $\vec{\kappa}, \vec{h}, \vec{C}, \vec{D}, \sigma \in M_{0}$. Let $E=\left\{\gamma \in \omega_{\omega_{2}+1}: M_{\gamma} \cap \omega_{\omega_{2}+1} \in\right.$ $\left.\omega_{\omega_{2}+1}\right\}$. Since this is a closed unbounded subset of $\omega_{\omega_{2}+1}$, there must be an ordinal $\gamma$ such that the set $C_{\gamma} \subset \gamma$ is cofinal of ordertype $\omega \cdot \omega \cdot \alpha$ and $C_{\gamma} \subset E$.

Also, fix a continuous increasing $\in$-tower $\left\langle N_{\delta}: \delta \in \omega_{2}\right\rangle$ of elementary submodels of large enough structure, each of them of size $\aleph_{1}$ and such that $N_{\delta} \cap \omega_{2} \in \omega_{2}$. In particular, $\vec{\kappa}, \vec{h}, \vec{C}, \vec{D}, E, \sigma, \gamma \in N_{0}$. Let $F=\left\{\delta \in \omega_{2}: N_{\delta} \cap \omega_{2}=\delta\right\}$. Since this is a closed unbounded set, there must be an ordinal $\delta \in \omega_{2}$ such that the set $D_{\delta} \subset \delta$ is cofinal of ordertype $\omega \cdot \omega \cdot \alpha$ and $D_{\delta} \subset F$.

We claim that the sequence $\vec{r}_{\gamma \delta}$ is a legal counterplay against the strategy $\sigma$. Well, consider the situation at round $\omega \cdot \beta+n$ for some ordinal $\beta \in \alpha$ and number $n \in \omega$. Suppose that up to this point, the sequence constituted a legal partial play; we want to see that it will provide a legal answer even in this round. The strategy $\sigma$ commands Adam to play partial Borel functions $\left\{f_{m k}: k \in n+1, m \in \omega\right\}$ such that for each $k \in n+1$ and each $m \in \omega$ the function maps $\left(2^{\omega}\right)^{n+1 \backslash\{k\}}$ to $\left(2^{\omega}\right)$. We must show that the $n+1$-tuple $\vec{t}=\vec{r}_{\gamma \delta}(\omega \cdot \beta+n) \in\left(2^{\omega}\right)^{n+1}$ is not on the graph of any of these functions, that is $\vec{t}(k) \neq f_{m k}(\vec{t} \uparrow(n+1 \backslash\{k\}))$.

To this end, fix integers $k \in n+1$ and $m \in \omega$. Define a function $g \in \prod_{\eta} \kappa_{\eta}$ by letting $g(\eta)$ to be the supremum of the set $\left\{\xi \in \kappa_{\eta}: s_{\xi}=f_{m k}(\vec{u}, \vec{v})\right.$, where $\vec{u} \in\left(2^{\omega}\right)^{k}$ is a sequence all of whose entries are on the $\vec{s}$-sequence, and are 
indexed by ordinals $<\sup \left\{\kappa_{\eta^{\prime}}: \eta^{\prime} \in \eta\right\}$, and $\vec{v} \in\left(2^{\omega}\right)^{(n+1) \backslash(k+1)}$ is a sequence all of whose entries are on the $\vec{s}$-sequence and are indexed in the ordinals in the range of the functions $\left.\left\{h_{\gamma^{\prime}}: \gamma^{\prime} \in C_{\gamma} \cap C(\gamma, \omega \cdot(\omega \cdot \beta+n)+k)\right\}\right\}$. It is immediate that this set has size $<\kappa_{\eta}$ and so the function $g$ is well-defined. There are two important points.

1. $g \in M_{C(\gamma, \omega \cdot(\omega \cdot \beta+n)+k)}$. This so happens because the function is defined from objects contained in the model, in particular from the set $C_{\gamma} \cap \gamma(\omega \cdot(\omega \cdot$ $\beta+n)+k$ ) which belongs to the model by the coherence of the $C$-sequence.

2. $g \in N_{D(\delta, \omega \cdot(\omega \cdot \beta+n)+k)}$ by the same reason as in the previous item, this time using the coherence of the $D$-sequence.

By the first point, the function $g \in \prod_{\delta} \kappa_{\delta}$ is dominated by the function $h_{C(\gamma, \omega \cdot(\omega \cdot \beta+n)+k)}$ from some ordinal on. By the second point, this ordinal must be smaller than $D(\delta, \omega \cdot(\omega \cdot \beta+n)+k)$. By the definition of the function $g$ and the sequence $\vec{t}=\vec{r}_{\gamma \delta}(\omega \cdot \beta+n)$ then, it must be the case that $\vec{t}(k) \neq f_{m k}(\vec{t} \uparrow$ $(n+1 \backslash\{k\}))$ as desired. The lemma follows.

So we have the following. Let $\vec{J}$ be the $\omega$-sequence of ideals given by $\vec{J}(n)=$ $J_{n+1}$.

Corollary 3.7. Let $\alpha$ be a countable ordinal. $\operatorname{non}\left(\vec{J}^{\alpha}\right) \leq \aleph_{\omega_{2}}+1$.

Let $K_{\omega}$ be the $\sigma$-ideal on $\left(2^{\omega}\right)^{\omega} \sigma$-generated by the graphs of partial Borel functions from $\left(2^{\omega}\right)^{\omega \backslash\{n\}}$ to $2^{\omega}$, for all numbers $n \in \omega$. The following lemma is an observation complementary to the previous results in this section. It shows that the ideal $K_{\omega}$ is different from the ideals $K_{n}$ in that its uniformity can be arbitrarily large:

Lemma 3.8. $\mathfrak{p} \leq \operatorname{non}\left(\mathrm{K}_{\omega}\right)$.

Proof. Suppose that $A \subset\left(2^{\omega}\right)^{\omega}$ is a set of size $<\mathfrak{p}$. We must produce partial Borel functions $\left\{f_{n}: n \in \omega\right\}$ such that $f_{n}:\left(2^{\omega}\right)^{\omega \backslash\{n\}} \rightarrow 2^{\omega}$ and every point in the set $A$ is on the graph of one of them. First, use the Axiom of Choice to find a set $B \subset A$ so that

1. for all sequences $\vec{x}, \vec{y} \in B$ if $\vec{x}(m)=\vec{y}(m)$ for all but finitely many integers $m$ then $\vec{x}=\vec{y}$

2. $B \subset A$ is a maximal set with the previous property.

For each number $n$ let $g_{n}$ be a partial function from $\left(2^{\omega}\right)^{\omega \backslash\{n\}}$ to $2^{\omega}$ defined by $g_{n}(\vec{x})=z$ if the sequence $\vec{y}_{0}=\vec{x} \cup\{\langle n, z\rangle\}$ is in the set $A$ and for some sequence $\vec{y}_{1}$ in the set $B, \vec{y}_{0}(m)=\vec{y}_{1}(m)$ for all numbers $m \geq n$. By the property (1) of the set $B$, this formula does correctly define the functions $g_{n}$, and by the property (2), every point in the set $A$ is on the graph of all but finitely many of these functions. Now there is a general fact, proved for example in [9] Appendix $\mathrm{B}$, that every partial function of size $<\mathfrak{p}$ between Polish spaces is a subset of a Borel function. Thus for every number $n$ there is a Borel function $f_{n}$ such that $g_{n} \subset f_{n}$; clearly every point in the set $A$ is on the graph of all but finitely many of the functions $f_{n}$ as desired. 
To prove the abovementioned general fact, consider for simplicity a partial function $g: 2^{\omega} \rightarrow 2^{\omega}$. By a standard almost disjoint coding argument find a $\sigma$-centered forcing adding subsets $\dot{a}_{i}: i \in \omega$ of $2^{<\omega}$ such that $x \cap \dot{a}_{i}$ is finite if and only $g(x)(i)=0$ for every infinite binary sequence $x$ in the ground model, viewed as the set of all its initial segments and so a subset of $2^{<\omega}$. By the small size of the function $g$ and Bell's theorem there will be sets $a_{i}: i \in \omega$ already in the ground model such that the Borel function $f: 2^{\omega} \rightarrow 2^{\omega}$ defined by $f(x)(i)=0$ iff $x \cap a_{i}$ is finite, extends the function $g$ as desired.

\section{The duality theorems}

The proof of Theorem 1.2 follows the argument for the duality theorem in the Applications section of [9]. Suppose that $n>0$ is a natural number and $I$ is an analytic ideal on the reals such that ZFC proves $\operatorname{cov}(\mathrm{I}) \geq \mathfrak{h m}_{n}$. We want to argue that non(I) $\leq \aleph_{n+1}$. For this, it is necessary to analyse the countable support iteration of the $P_{n}$ forcing. The following Facts use the terminology and arguments from [9]. Recall:

Definition 4.1. [9] Suppose $K$ is a $\sigma$-ideal on the reals and $\alpha \in \omega_{1}$ is an ordinal. A set $B \subset \mathbb{R}^{\alpha}$ is $K$-perfect if the tree $T \subset \mathbb{R}^{\leq \alpha}$ of all initial segments of the sequences in the set $B$ has the following two properties. It is $K$-positively branching, meaning that for every node $t \in T$ the set $\left\{r \in \mathbb{R}: t^{\frown} r \in T\right\}$ is not in the ideal $K$. And it is $\sigma$-closed, meaning that for every sequence $t_{0} \subset t_{1} \subset \ldots$ of sequences in the tree $T$ we have $\bigcup_{n \in \omega} t_{n} \in T$.

Fact 4.2. For every countable ordinal $\alpha \in \omega_{1}$ and every analytic set $A \subset\left(2^{\omega}\right)^{\alpha}$, either the set $A$ contains a Borel $J_{n}$-perfect subset, or it belongs to the ideal $J_{n}^{\alpha}$. Under the assumption of the existence of $\omega$ many Woodin cardinals, this extends to all projective sets.

This is an immediate consequence of Lemmas 2.1 and 2.2, Corollary 2.5 and the work of [9].

Fact 4.3. For every countable ordinal $\alpha \in \omega_{1}$ the ideal $J_{n}^{\alpha}$ is homogeneous.

This happens because the ideal $J_{n}$ is homogeneous. It means in particular that for every Borel $J_{n}$-perfect set $B \subset\left(2^{\omega}\right)^{\alpha}$ the invariant non $\left(J_{n}^{\alpha}\right)$ is equal to $\operatorname{non}\left(\mathrm{J}_{n}^{\alpha} \uparrow \mathrm{B}\right)$, the smallest size of a $J_{n}^{\alpha}$-positive subset of $B$.

Fact 4.4. There is a countable ordinal $\alpha \in \omega_{1}$, a Borel $J_{n}$-perfect subset $B \subset$ $\left(2^{\omega}\right)^{\alpha}$ and a Borel function $f: B \rightarrow \mathbb{R}$ such that $f$-preimages of I-small sets are $J_{n}^{\alpha}$-small.

This is true because after the countable support iteration of the $P_{n}$-forcing of length $\mathfrak{c}^{+}$, it is still the case that $\operatorname{cov}(\mathrm{I}) \geq \mathfrak{h m}_{n}$ and so there must be a name for a real which falls out of all ground model coded $I$-small sets. This name must be represented by a Borel set and a function as in the above Fact-[9].

Now fix $\alpha, B$ and $f$ from the previous Fact. By Fact 2.3 and Corollary 3.4, there is a $J_{n}^{\alpha}$-positive subset $A \subset B$ of size $\leq \aleph_{n+1}$. By the properties of the 
function $f$, the set $f^{\prime \prime} A$ must be an $I$-positive set of size $\leq \aleph_{n+1}$, and Theorem 1.2 follows.

The argument for Theorem 1.3 is similar, using the analysis of the countable support iteration in which the forcings $P_{n}$ alternate and Corollary 3.7. Theorem 1.1 is just a special case of Theorem 1.2.

\section{References}

[1] T. Jech, Set theory, Pure and Applied Mathematics. Academic Press, New York-London, 1978.

[2] A. Kechris, On a notion of smallness for subsets of the Baire space, Trans. Amer. Math. Soc. 229 (1977), 191-207.

[3] D. A. Martin, A purely inductive proof of Borel determinacy, Recursion theory (Ithaca, NY, 1982), 303-308, Proc. Sympos. Pure Math., 42, Amer. Math. Soc., Providence, RI, 1985.

[4] S. Shelah, Analytical guide and updates for "Cardinal arithmetic," Shelah [Sh:E12].

[5] _ Non-structure theory, to be published by Oxford University Press.

[6] __ Cardinal arithmetic, Oxford Logic Guides, 29. Oxford Science Publications. The Clarendon Press, Oxford University Press, New York, 1994.

[7] S. Geschke, M. Goldstern, M. Kojman, Continuous ramsey theory and covering the plane by functions, preprint, 2001.

[8] S. Geschke, M. Kojman, W. Kubiś, R. Schipperus, Convex decompositions in the plane and continuous pair colorings of the irrationals, preprint, 2001.

[9] J. Zapletal, Cardinal invariants and descriptive set theory, preprint, 2002.

[10] Forcing with ideals of closed sets, Comment. Math. Univ. Carolinae 43 (2002), $181-188$.

Einstein Institute of Mathematics, Hebrew University, Jerusalem 91904, Israel.

E-mail address: shelah@math.huji.ac.il

358 Little Hall, P.O. Box 118105, Gainesville FL 32611, U.S.A.

E-mail address: zapletal@math.ufl.edu 